\title{
Pathogenesis of pupillary capture after posterior chamber intraocular lens implantation
}

\author{
MICHAEL LAVIN AND JONATHAN JAGGER
}

From the Western Ophthalmic Hospital, London

SUMmary Pupillary capture is an unusual complication of posterior chamber intraocular lens implantation and may occur in the early or late postoperative period. We describe a series of patients with early or late onset pupillary capture and describe the pathogenesis. Early capture results from mechanical factors and is usually preventable. Late capture may be mechanical but is usually a sequel to the formation and subsequent contraction of iridocapsular synechiae. Synechiae result from lens epithelial or iris stromal hyperplasia and myofibroblastic metaplasia; they may be minimised by an atraumatic technique and adequate control of postoperative inflammation. The effect of pupillary capture is largely cosmetic, and we encountered no specific complications attributable to it.

Posterior chamber intraocular lenses (IOLs) are the most common type of IOL inserted at cataract extraction in the USA.' An uncommon complication is pupillary capture or entrapment. Pupillary capture (PC) occurs when part of the pupil margin is displaced posteriorly behind the IOL optic, which then appears to lie in the anterior chamber. ${ }^{2}$

PC is more frequently described as an early event, although a late form is recognised. ${ }^{2-6}$ Early PC is attributed to mechanical factors, while late PC is not adequately explained. A variety of pharmacological and surgical corrective manoeuvres have been described. ${ }^{240-8}$

We have seen a number of patients with either early or late PC. All patients had normal pseudophakic anatomy at the completion of surgery, but developed PC at intervals ranging from one day to two months postoperatively. In this paper we report our findings and relate them to the pathophysiology of the eye after extracapsular cataract extraction and posterior chamber IOL implantation.

\section{Materials and methods}

We performed a prospective study of pupil and IOL relationships in patients undergoing routine extracapsular cataract extraction (ECCE) and IOL implantation. All IOLs had anteriorly angled

Correspondence to J Jagger, FRCS, Moorfields Eye Hospital, City Road, London EC1V 2PD haptics. All patients had been noted to have normal pseudophakic anatomy at the conclusion of surgery. In the postoperative period any uveitis was controlled with intensive topical dexamethasone before discharge. Patients were included in the study if PC was diagnosed in the immediate postoperative period or at follow-up. PC was classified as early if diagnosed in the first five days after surgery and as late if diagnosed later.

\section{Results}

Fifteen patients with $\mathrm{PC}$ of a posterior chamber IOL were identified. The time of onset ranged from 1 to 60 days postoperatively, mean 30 days. The patients ranged in age from 51 to 85 years, mean 69 years. Eight patients were female. These patients were seen during a period when 480 ECCE and IOL implantations were performed, yielding an incidence of $\mathrm{PC}$ of $3 \cdot 1 \%$. All cases of PC have been seen in patients with ciliary sulcus fixated posterior chamber IOLs. To date no patient with an 'in the bag' posterior chamber IOL has developed PC.

Two patients suffered early PC; in one of them it was reversed but late $\mathrm{PC}$ subsequently developed. The second patient with early PC did not respond to initial intensive pharmacological attempts at reversal, but the condition spontaneously reversed three months later, with no evidence of residual posterior synechiae formation. 
Fourteen patients suffered late PC. At diagnosis of late PC large numbers of pigmented deposits on the IOL were frequently observed, and many patients had a persistent iritis. No complications attributable to the PC were seen.

Patients with late PC had many factors associated with ocular inflammation and iris trauma (Table 1), and only one patient had an unremarkable course. Thirteen of these patients had late PC resulting from the formation of iridocapsular synechiae with displacement of the pupil margin. These iridocapsular synechiae were found to be continuous with and adherent to zones of posterior capsular opacity. One patient suffered late reversible mechanical PC.

Pre-existing ocular diseases included glaucoma in two patients and single patients with prior excision of iris melanoma and broad iridectomy, diabetic maculopathy, senile macular degeneration, and amblyopia.

\section{ILLUSTRATIVE CASES}

Case 1. A 74-year-old Indian male on treatment for chronic open angle glaucoma underwent routine ECCE and posterior chamber IOL implantation by a junior staff member. On the first postoperative day PC was diagnosed and treated successfully with initial pharmacological mydriasis and later miosis. At discharge four days later the pupil was free, with no evidence of PC. The pupil margin remained free at initial follow-up visits. The patient developed a marked postoperative uveitis with questionable compliance, and by two months postoperatively had developed synechial pupil capture.

Case 2. A 60-year-old West Indian female had previously undergone trabeculectomy for glaucoma and had residual posterior synechiae. A broad iridec-

\section{Table 1 Predisposing factors in 14 patients with late pupil} capture

\begin{tabular}{lll}
\hline Preoperative: & Prior iritis & 4 \\
& Posterior synechiae* & 4 \\
& Prior surgery† & 2 \\
Operative: & Difficult surgery $\ddagger$ & 6 \\
& Broad iridectomy§ & 4 \\
& Peripheral iridectomy & 3 \\
Postoperative: & Severe postoperative iritis & 6 \\
& Poor compliance & 5 \\
& Wound leakage & 2 \\
& Acute PC & 1 \\
& Uneventful course & 1
\end{tabular}

*Three due to prior iritis, one to prior trabeculectomy.

†One trabeculectomy; one iris melanoma excised three years previously.

$\ddagger$ Includes cases with intraoperative iris trauma, and those where technical difficulties in expressing the nucleus, aspirating cortex, maintaining the anterior chamber, or inserting IOL were encountered.

§Three intraoperative; one three years previously for iris melanoma. tomy with ECCE and IOL implantation were performed, with normal pupil-IOL relationships in the early postoperative period and at first follow-up visit. The patient did not comply with treatment and presented four weeks after surgery with a marked iritis and posterior iridocapsular synechiae formation behind the IOL, forming PC.

Case 3. A 60-year-old Caucasian was seen two weeks after routine ECCE and IOL implantation for a Nd-YAG laser capsulotomy. The IOL position was normal, and the pupil was then dilated. Spontaneous pupil capture developed, but was subsequently reversed with topical thymoxamine. No further episodes of PC were observed.

\section{Discussion}

Early onset PC is the result of mechanical factors such as wound leakage with shallow anterior chamber, pupillary dilatation, air in the anterior chamber forcing the iris behind the IOL, or reversal of IOL, and is more frequently seen in IOLs with planar haptics than other types. ${ }^{2-7}$ Mechanical factors appear to be responsible for all cases of early PC, but may cause recurrent or even late PC (case 3 ). This form of PC results from movement of the free pupil margin behind the IOL optic owing to the relative anterior displacement of the IOL, or the relative posterior displacement of the pupil margin. Subsequent synechiae formation may preclude nonsurgical reversal. Of our two cases of early mechanical PC one was reversed by mydriatics while the other was not. In the latter patient the condition subsequently reversed spontaneously. Reversal manoeuvres that have been described include mydriasis with external limbal haptic massage and subsequent miosis, ${ }^{24}$ Nd-YAG laser-induced mechanical shock-wave lens retropulsion, ${ }^{8}$ and direct surgical manipulation..$^{5}$ Other authors advocate no intervention. ${ }^{346}$ Our patients suffered no apparent ill effects of early PC.

Late PC is seen two weeks to nine months postoperatively (Pearce J, personal communication). Although it may occasionally be mechanical in origin (case 3), it is more frequently synechial in origin. Our observations indicate this latter type to be the result of iridocapsular synechiae formation about the edge of the IOL optic, with subsequent contraction pulling the iris posteriorly (Fig. 1). Several changes in the early postoperative period contribute to synechiae formation between the iris margin and the posterior capsule. Immediately after ECCE fibrinous strands extend from the anterior capsule edges towards the posterior capsule, with which they subsequently make contact. ${ }^{9}$ Remnants of the anterior capsular lens epithelium proliferate in response to factors 


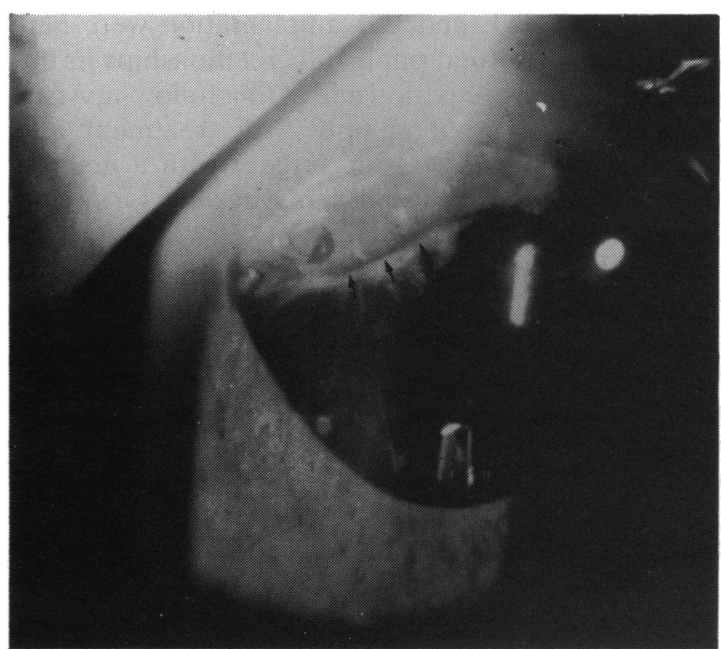

Fig. 1 Synechial pupil capture with posterior capsular striae and opacification. Arrows delineate iridocapsular synechiae behind IOL.

present in the aqueous of injured eyes. ${ }^{10}$ These proliferating lens cells extend on to the posterior capsule, where they spread and undergo myofibroblastic metaplasia. ${ }^{12}$ This process, resulting in a variety of fibrous posterior capsular opacities, is more extensive and severe in younger patients, who have larger numbers of active lens cells, and may

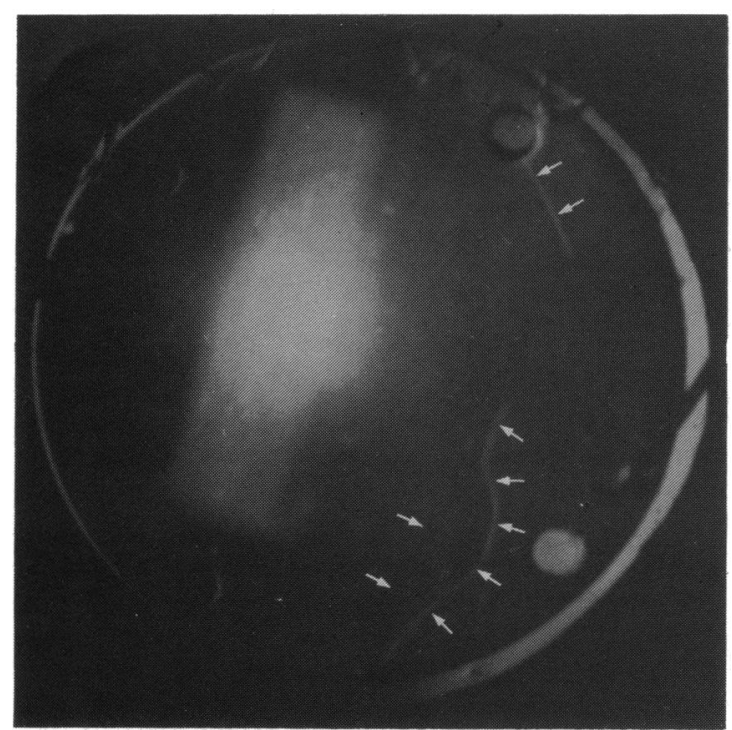

Fig. 2 Appearance of free anterior capsular flaps between $I O L$ and posterior capsule six months after secondary $I O L$ implantation. Note clear edges of anterior capsule remnants, with absence of fibrosis or opacification.

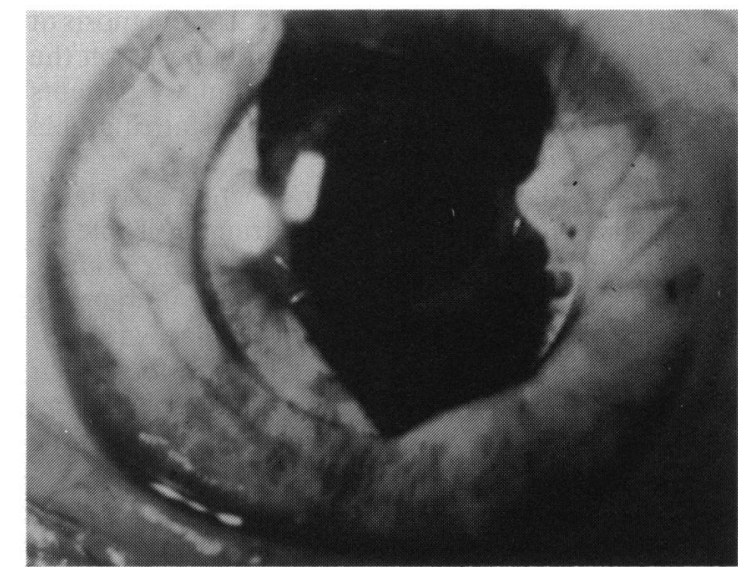

Fig. 3 Synechial pupil capture following ECCE, broad iridectomy, and inferior sphincterotomy in a patient with prior iritis and extensive posterior synechiae. Iridocapsular synechiae (arrows) are continuous with posterior capsular opacity.

opacify the visual axis. ${ }^{112}$ In older patients, with fewer viable lens cells and less lens epithelial metabolic activity, the process is largely limited to the area of anterior to posterior capsule contact. ${ }^{.1}$ The importance of viable lens epithelium in the process of fibrous metaplasia is illustrated by Fig. 2, the case of an elderly male who had undergone ECCE for a morgagnian cataract. In such a cataract the lens epithelial cells are necrotic, and fibrous metaplasia from this source cannot occur. In neither the early nor late postoperative period did this patient develop synechiae, and both his anterior capsule remnants and posterior capsule remained entirely free and clear at 12 months after ECCE and even six months after secondary posterior chamber IOL implantation.

Fibrous metaplasia in the anterior segment does not originate only from lens cells, but may also occur from inflamed or traumatised iris tissue..$^{1314}$ Iritis of any origin is frequently accompanied by the formation of synechial adhesions. In experimental ECCE uveal cells are observed to form bridges from the anterior uveal epithelium to the anterior and posterior capsule. ${ }^{13}$ Our clinical observations support these experimental findings. In our patients iris and pupil margin trauma were a prominent feature (Fig. 3 ). This indicates that the presence of iris trauma, with iris cell release, contributes to the process of synechiae formation between the pupil margin and the posterior capsule round the IOL optic. It is noteworthy that uveal cells have been found to form a significant component of the posterior capsular opacification seen after experimental ECCE.${ }^{13}$ Cells of iris origin, in association with lens epithelial cells, 
play an important part in the formation of both iridocapsular synechiae and of posterior capsular opacification. Reduction of iris manipulation and trauma during ECCE may be important in preventing both pupil and posterior capsule complications. While some surgeons advocate resuturing broad iridectomies, it is not likely that this would reduce the total number of uveal cells released into the anterior chamber, but may mechanically keep the iris plane more anterior.

The question arises whether the IOL itself may directly trigger some of the processes involved in PC. Macrophages are deposited on the surfaces of most IOLs soon after implantation as part of a foreign body reaction. ${ }^{15-17}$ These cells can subsequently undergo metaplasia to fibroblast-like cells ${ }^{16}$ and may contribute to synechiae formation about the IOL edges, particularly in the presence of iris trauma or inflammation. While macrophages may contribute, it appears that posterior iridocapsular synechiae arise largely from iris and lens epithelial myofibroblastic metaplasia.

We found late PC to be most commonly a synechial event resulting from iridocapsular synechiae, with subsequent contraction drawing the pupil margin posteriorly. In a histopathological study of eyes at necropsy, posterior iridocapsular or iridovitreal synechiae were observed in $14 \%$ of eyes with posterior chamber IOLs. ${ }^{18}$ In our patients severe iritis or iris trauma were noteworthy risk factors. At the time of diagnosis of PC the IOLs frequently had large numbers of pigmented deposits which have been shown to be macrophages. ${ }^{17}$ In several patients these deposits have made posterior capsulotomy with the Nd-YAG laser more difficult. In addition, because these patients have a fixed pupil, fundus examination and treatment is compromised. We have not observed any other complications of synechial PC.

In conclusion, acute PC is usually due to mechanical factors which may be avoided by scrupulous attention to surgical detail. Late PC may occasionally be a reversible mechanical phenomenon, but is more commonly a sequel to iridocapsular synechiae formation and contraction. Synechial PC is more often seen after difficult surgery, iris trauma, severe iritis, or poor patient compliance.

We thank Mr Ron Marsh and Mr Redmond Smith for access to patients under their care and for helpful comments, and Ms Suc Ford for photographic assistance.

\section{References}

1 Stark WJ, Terry AC, Worthen D, Murray GC. Update of intraocular lenses implanted in the United States. $A m J$ Ophthalmol 1984; 98: 238-44.

2 Lindstrom RL, Hermann WK. Pupil capture: prevention and management. Am Intraocul Implant Soc J 1983; 9: 201-4.

3 Lim AS. A colour atlas of posterior chamber implants. Bristol: Wright, 1985.

4 Abrahamson IA, Stein LN. Pupillary entrapment-a postoperative complication with PC-IOLs. Cataract 1984; 1: 10-2.

5 Lippman JI. Pupillary abnormalitics associated with posterior chamber lens implantation. Ophthalmic Surg 1982; 13: 197-200.

6 Jaffe NS. Cataract surgery. 4th ed. St Louis: Mosby, 1984: 223-5.

7 Thrasher BH. Extracapsular cataract extraction with (Shearing) lens implantation technique. In: Rosen ES, Haining WM, Arnott EJ, eds. Intraocular lens implantation. St Louis: Mosby, 1984: 417-23.

8 Steinert RF, Puliafito IA. The Nd: YAG laser in ophthalmology. Philadelphia: Saunders, 1985.

9 Smith RJH, Doran R, Caswell A. Extracapsular cataract extraction-some problems. Br J Ophthalmol 1982; 66: 183-5.

10 Weinsieder A, Reddan J, Wilson D. Aqueous humour in lens repair and cell proliferation. Exp Eye Res 1976; 23: 355-63.

11 Sakanishi Y, Sawa M, Shimizu H. Clinical classification of aftercataract following planned extracapsular cataract extraction. Jpn J Ophthalmol 1984; 28: 299-309.

12 McDonnell PJ, Zarbin MA, Green WR. Posterior capsule opacification in pseudophakic eyes. Ophthalmology (Rochester) 1983; 90: 1548-53.

13 Odrich MG, Hall SJ, Worgul BV, Trokel SL, Rini FJ. Posterior capsule opacification: experimental analyses. Ophthalmic Res 1985; 17: 75-84.

14 Yeo YH, Jakobiec FA, Pokorny K, Iwamoto T, Piscano A. The ultrastructure of a 'cocoon membranc'. Ophthalmology (Rochester) 1983; 90: 410-9.

15 Wolter JR, Felt DP. Proliferation of fibroblast-like cells on failing intraocular lenses. Ophthalmic Surg 1983; 14: 57-64.

16 Wolter JR. Cytopathology of intraocular lens implantation. Ophthalmology (Rochester) 1985; 92: 135-42.

17 Ohara K. Biomicroscopy of surface deposits resembling forcignbody giant cells on implanted intraocular lenses. $A m J$ Ophthalmol 1985; 99: 304-11.

18 Champion R, McDonnell PJ, Green WR. Intraocular lenses. Histopathologic characteristics of a large series of autopsy cyes. Surv Ophthalmol 1985; 30: 1-32.

Accepted for publication 3 March 1986. 